\title{
Evolving Concept of Cancer Stem Cells: Role of Micro-RNAs and their Implications in Tumor Aggressiveness
}

\author{
Seema Sethi and Fazlul H. Sarkar*
}

Department of Pathology, Karmanos Cancer Institute, Wayne State University School of Medicine, Detroit, MI,USA

\begin{abstract}
Recent advances in cancer stem cell (CSC) research and the role of miroRNAs (miRNAs) provided renewed interest, as supported by some convincing data showing that deregulation of miRNAs are important molecular events in the maintenance of CSC niche, which contribute to therapeutic resistance, resulting in treatment failure. Therefore, we are succinctly providing state-of-the-art knowledge on miRNAs that are associated with CSCs in this article, and it is quite clear that CSCs and miRNAs are implicated in therapeutic resistance, suggesting that novel approaches must be devised to eliminate CSCs or drug resistant cells focusing on overcoming therapeutic resistance. This indeed could be achieved by targeted strategies either to re-express the lost miRNAs or inactivate over-expressed miRNAs in cancers in general and CSCs in particular. We sincerely hope that this article would be educational to attract innovative research in the field so that we can find specific novel approaches to eradicate cancer by overcoming therapeutic resistance by targeting miRNAs, which will be useful to eliminate tumor recurrence and metastasis.
\end{abstract}

\section{General Introduction of the Topic}

There is an evolving concept that cancers are diseases driven by cancer stem cells (CSCs) [1]. These are a small number of undifferentiated cells resembling primitive cells present within the tumor mass that have self-renewal and tumorigenic potential [2]. This unique capability allows them to self-renew, differentiate and form cells which are phenotypically, and most often genotypically similar to the parent cancer cells [3]. Eventually they lead to tumor re-growth with invasive and metastatic propensities [2]. This concept is supported by the fact that most tumors are a heterogenous admixture of cells in varying stages of differentiation with the tumor initiating cells comprising only a small percentage of the entire tumor cell population [4-6]. These cells have been demonstrated in human tumor specimens, and both in vitro cell cultures models and animal model studies of human tumors using severe combined immunodeficient (SCID) mice [7]. In the following paragraphs we will succinctly summarize the concept of CSCs and miRNAs with respect to therapeutic resistance.

\section{Relevance of Cancer Stem Cells (Cscs) in Tumors}

Although the CSC concept was proposed several decades ago it is recently gaining impetus due to its emerging clinical and translational relevance. CSCs are being implicated not only in tumor initiation, but also in tumor progression and disease relapse after treatment. As a result of this, they impact patient management protocols, as their presence is associated with poor clinical outcomes [6] and drug resistance [8] ultimately resulting in tumor invasion and metastasis.

In recent studies the significance of CSCs leading to drug resistance in patients on chemotherapeutic drugs has been emphasized and described to be an acquired phenomenon [8]. It is generally seen that patients initially show good response to an accepted and approved therapeutic drug regimen but suddenly worsen due to tumor unresponsiveness. The occurrence or enrichment of CSCs eventually impacts the morbidity and mortality of patients due to tumor re-growth and metastases. This has grave consequences in the clinical scenario because the presence of CSCs prevents the eradication of tumors [9], suggesting that novel approaches must be devised to eliminate CSCs in order to have complete eradication of tumors.

Evaluation of the CSC population in a patient shows additional promising clues which serve as potential markers to evaluate the clinical benefit of anti-tumor treatments. In the current clinical context, the effect of treatment on tumors is evaluated by a reduction in the size of the tumor. However, this is not necessarily indicative of improvement in long term survival of patients [10]. In fact, recently it has been proposed that response and survival rates may be independent entities reflecting treatment effects against distinct cancer cell types [6]. Hence it has been suggested that the short term tumor size reduction could reflect the effect of treatment on the bulk of the tumor cells; where as the long term survival benefit could be due to the effect of the treatment on the CSC's [6] which are known to be highly resistant to conventional therapeutics.

\section{Strategies for the Identification of CSC's}

CSCs can be identified in tumors using immunohistochemistry by targeting specific cell surface markers. Cell surface markers are molecules located on the surface of cells which can be targeted using immunohistochemical approach. Once the cell surface marker finds its complementary strand in the in the immune complex, the color in the chromogens is seen microscopically to identify the lineage of the cells. Since CSCs are tiny cells with no phenotypic morphologic features of the parent cell of origin, these cell surface markers are specifically helpful in the clinical context in identifying the lineage to which CSC's belong. They form the basis of the clinically useful immunohistochemical approach, for identifying CSCs. Their nomenclature is based on Cluster Designation (CD).

*Corresponding author: Fazlul H. Sarkar, Department of Pathology, Barbara Ann Karmanos Cancer Institute, Wayne State University School of Medicine, 740 Hudson Webber Cancer Research Center, 4100 John R, Detroit, MI 48201, USA Tel: 313-576-8327; Fax: 313-576-8389; E-mail: fsarkar@med.wayne.edu

Received May 24, 2011; Accepted June 15, 2011; Published June 17, 2011

Citation: Sethi S, Sarkar FH (2011) Evolving Concept of Cancer Stem Cells: Role of Micro-RNAs and their Implications in Tumor Aggressiveness. J Carcinogene Mutagene S1:005. doi:10.4172/2157-2518.S1-005

Copyright: (C) 2011 Sethi S, et al. This is an open-access article distributed unde the terms of the Creative Commons Attribution License, which permits unrestricted use, distribution, and reproduction in any medium, provided the original author and source are credited. 
The expression levels of these cell surface markers can be used to identify CSCs. AML CSC's residing in the endosteal region of the bone marrow are CD34 positive and CD38 negative [11,12]. CML stem cells are also CD34 positive [13]. The cell surface marker, CD133, identifies CSCs from colon cancer [14-16], glioblastomas [17,18], ovarian cancer $[19,20]$ and small-cell lung carcinomas [21,22]. Additionally, ovarian CSCs are also CD117 positive and some are CD44 positive and CD24 negative $[19,20]$. Small-cell lung carcinoma CSCs are ABCG2 positive or uPAR positive $[21,22]$. Osteosarcoma CSCs are CD117 positive, Stro-1 positive, CXCR4 positive and ABCG2 positive [23]. CD44 positivity has been observed in both pancreatic and breast cancer CSCs. Additionally, there is CD24 negativity in pancreatic cancer CSCs $[24,25]$ and CD24 low expression in breast cancer CSCs. High CD29 positivity $(+++)$ along with CD24 moderate positivity $(++)$ is also seen in breast cancer CSCs [26,27]. Hepatocellular carcinoma CSCs are Hoechst positive [28]. Hoechst positive cells are CSCs which are able to efflux the dye Hoechst 33342 through an ATP-binding cassette (ABC) membrane transporter. This is used for detecting CSCs in various cell lines. Prostate CSCs show high expression of CD117 and ABCG2 $[29,30]$.

CSCs can also be identified with the use of functional characteristics like aldehyde dehydrogenase (ALDH) activity and increased drug efflux potential [31-33]. ALDH1 has been used as a marker for breast CSCs and also has served as an indicator of poor prognosis [31], which is further discussed below.

\section{Cancer Stem Cells (Cscs) in Different Malignant Tumors Associated with Therapeutic Resistance}

CSCs have been identified and isolated from tumors of the hematopoietic system, breast, lung, prostate, colon, brain, head and neck, and pancreas [34]. They have been associated with chemotherapeutic drug-resistance in colorectal malignancies [1416], acute myelogenous leukemia (AML) [11,12], small-cell lung carcinomas [21,35] and osteosarcomas [23]. In breast tumors, CSCs contribute to cisplatin and paclitaxel resistance [26,27]. Chronic myelogenous leukemia CSCs are intrinsically resistant to imatinib [13]. In glioblastomas, CSCs show significant resistance to temozolomide, carboplatin, paclitaxel and etoposide [17]. In human hepatocellular carcinomas, resistance to doxorubicin or methotrexate has been implicated due to CSCs [28]. Pancreatic CSCs are gemcitabine-resistant [25]. Ovarian CSCs have been demonstrated to be markedly resistant to carboplatin and paclitaxel $[19,36]$ as well as to cisplatin [20]. Prostate CSCs are resistant to arsenic-induced cytolethality [29] and cisplatin, paclitaxel, doxorubicin and methotrexate [30]. These reports and many others clearly suggest that CSCs are broadly resistant to conventional therapeutics.

\section{Role Of Cscs in Prognosis}

Recent studies have demonstrated that CSCs can be used as predictors of clinical outcome in tumors by using either genetic signatures, phenotypic characteristics or by functional assays [6]. Tumors which have a poor outcome have either increased CSCs or their cells take on the CSC characteristics [6]. Presence of CSCs in malignancies has been indicative of poor prognosis, failure of anticancer therapies and reduced patient survival. This has been amply demonstrated by several studies. CD133 positive CSCs in glioblastomas are associated with worse progression-free and overall survival [18]. ALDH1 positive CSCs indicate poor clinical outcomes in breast [31] and pancreatic adenocarcinomas [37]. CD44 positive and CD24 negative breast CSCs are associated with increased distant metastasis [38], worse overall and metastasis-free survival in patients with localized disease and are linked to a panel of 186 genes of 'invasiveness' [39]. Similar 'invasive gene signatures' associated with poor prognosis are also seen in lung and prostate cancers and medulloblastomas [6]. Gene expression studies using microarrays have also demonstrated a similar association with poor prognosis with CD44 positive CD24 negative breast CSCs [40]. Here will now discuss the role of CSCs and therapeutic resistance.

\section{CSCs in radioresistance}

Breast and glioblastoma CSCs are relatively radioresistant as compared to the bulk of the tumor cells. This is primarily due to the ability of breast CSCs to enhance handling of reactive oxygen species to minimize DNA damage [41]. In glioblastoma CSCs there is increased activation of the DNA damage checkpoint response [42]. These reports are some of the examples; however, further research in this area is ongoing.

\section{CSCs in chemotherapeutic resistance}

An increase in CSCs (enrichment of CSCs) has been demonstrated after conventional chemotherapy in clinical studies [43] as well as in xenograft models [44] indicating their role in therapeutic resistance, which has been documented in previous paragraphs. Spheroid cultures are mechanisms by which one can enrich CSCs. This is done to evaluate stem cell activity in normal tissue and putative CSC, Non-adherent spheroid cultures are increasingly being used in this regard. In this the cells are grown on non-adherent surfaces and give rise to spheroids that have the capacity for self-renewal and can in principal generate all the cell types of the cell of origin. The capacity for repeated generation of spheres from single cells is generally viewed as evidence of selfrenewal. Recently, it was described that spheroid-derived cells (SDC) from malignant cell lines possess CSC capacity.

Currently most chemotherapeutic agents target the rapidly proliferating cells and spare the relatively quiescent CSCs. Due to this biological phenomenon, the bulk of the tumor cells are destroyed but the remaining CSCs are untouched and/or further enriched, which eventually leads to tumor re-growth. It has been observed that when drug sensitivities are tested for cancer cell progeny, often the therapeutic protocols fail to take into consideration the ability of the drugs to target the CSCs, and more importantly CSC-targeted drugs are not currently available. These observations clearly suggest that novel strategies must be devised for selective eradication of CSCs, which should be the most urgent objectives toward achieving cure for cancer, and such strategies must be devised focusing on overcoming therapeutic resistance. Although the molecular mechanism of therapeutic resistance is multifaceted, in the following sections we will briefly discuss some of the known molecular mechanism(s).

\section{Mechanisms of Therapeutic Resistance by CSCs}

The presence of CSCs in a tumor mass causes therapeutic resistance by several mechanisms including reducing the intracellular level of cytotoxic agents or by increasing the capacity to repair the effects of cytotoxic injury. This has been exemplified in several tumors. The multiple myeloma CSCs increase the membrane-bound drug transporters and increase the intracellular detoxification enzymes that mediate drug efflux and metabolism [45]. CML and NHL (mantle cell type) CSCs either decrease the expression of proteins or lead to drug resistance as a result of their quiescent nature, which leads to resistance 
especially for drugs that are dependent on cell cycle progression for their actions $[46,47]$.

Some CSCs induce mutations in the cancer cells due to long-term exposure to drug, cause over-expression of drug transporters and DNA repair enzymes and many of these processes are regulated via dysregulation of miRNAs [1,48-50], and are further discussed below.

\section{The Role of Mirnas and their Relevance to Cscs}

As indicated previously that miRNAs are recently identified novel small non-coding RNAs investigated in the last ten years which are known to be critically involved in CSC regulation. Despite their small size of only 19-25 nucleotides, these molecules have revolutionized the molecular paradigm of gene regulation [51]. They are actively involved in post-transcriptional regulation and control several key physiological and pathological processes including cancer [52]. In normal physiologic states miRNAs are expressed normally but in pathologic conditions like cancer, their expressions are altered. Thus miRNAs may provide a targeted approach in treating cancers. The therapeutic decision-making in clinical oncology is currently undergoing a transition towards personalized pharmaco-therapeutics and the treatment archetype is being driven more by molecular characteristics than the anatomic site of tumor origin, and thus miRNAs are gaining momentum in the clinical arena. Specific miRNAs have been identified to play important roles in the formation and regulation of CSCs [53] including many different cellular processes like differentiation, proliferation and apoptosis. Despite their small size (about 22 nucleotides) these noncoding RNAs have revolutionized the current paradigm of cancer gene regulation as summarized below.

Mechanistically, the miRNAs act via the messenger RNAs (mRNAs). The 5 'end of miRNA binds to complementary regions in the 3' untranslated region (UTR) of the target messenger RNAs (mRNAs) leading to either the degradation of mRNA or inhibition of its translation to proteins.

Several miRNAs have a prime role in the regulation of CSCs. For example, miRNA-200c suppresses the formation of ducts and tumors by breast CSCs [54] whereas miR-125b makes the CSCs insensitive to chemotherapy [1] and regulates the proliferation of glioma CSCs [55]. It has been reported that ZEB1 could directly control the expression of miR-200, miR-203 and miR-183 and that knockdown of ZEB1 reduces pancreatic CSCs [56]. Several other miRNAs are very important in certain cancers; for example miR-34 is involved in pancreatic CSCs self-renewal [57], and miR-30 is known to inhibit self-renewal of breast CSCs [58]; and thus its over-expression reduces tumorigenesis and lung metastasis in mice, and by blocking its expression enhances tumorigenesis and metastasis [58]. Another example is the miR-1719 family where increased miR-17-19b has been shown to reduce differentiation and increased proliferation of leukemia CSCs [58] whereas miR-181 is important for the maintenance of hepatic CSC's [57]. The target information for each miRNA may be accessed by the readers from the link http://www.targetscan.org/ http://www. microrna.org/microrna/home.do. The role of miRNAs in relation to CSCs and cancer has become an emerging area of active research, which is highly likely to bear fruits in the targeted treatment of human malignancies and other chronic diseases. In the next section, we further catalogue some important observations as to the role of miRNAs in CSCs (summarized in Figure 1).

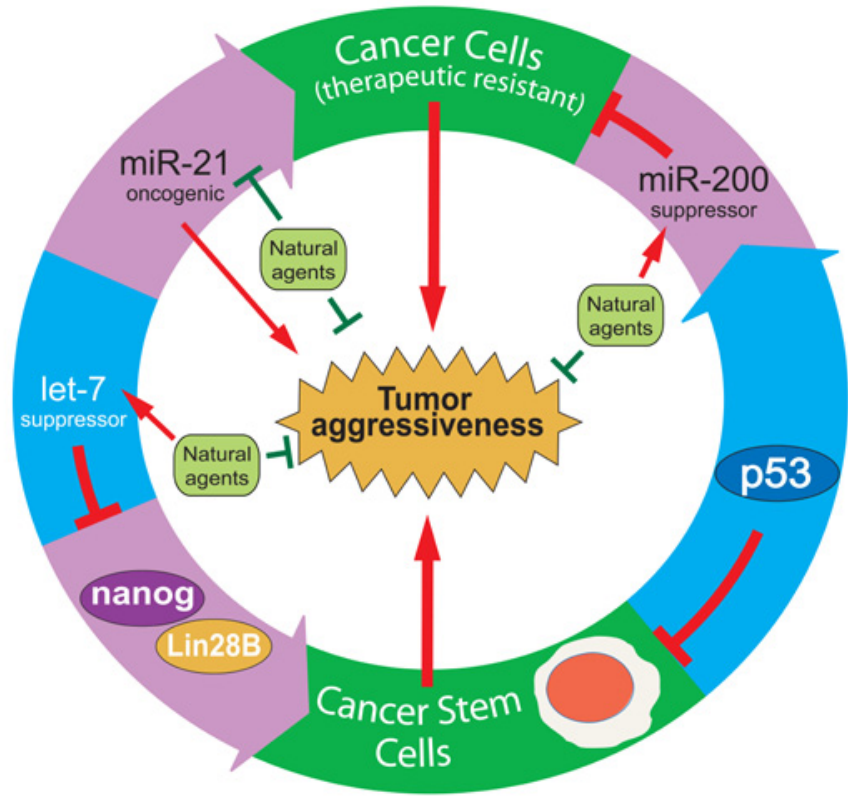

Figure 1: A simplified example on the relationship between CSCs and miRNAs with tumor aggressiveness. The miRNA miR-21 is over-expressed in cancers contributing to increased tumor aggressiveness while let-7 miRNA has tumor suppressor effect leading to reduction in tumor aggressiveness, and accumulation of let-7 is prevented by Lin28B, which is over-expressed in many cancers. Moreover, p53 activates miRNA miR-200 to suppress EMT related CSC properties, ultimately leading to reduced tumor aggressiveness whereas natural agents target CSCs and reduce tumor aggressiveness by down-regulating $\mathrm{miR}-21$ and up-regulating miR-200 and let-7.

\section{The Role of Specific Mirnas in Human Malignancies Associated with CSCs}

It has been well described that miRNAs are aberrantly expressed in human malignancies and regulate cancer progression [52], metastasis [59] and tumor aggressiveness, in addition to forming and regulating CSC's [53]. These molecules are clinically significant since miRNAs have the potential to be clinically useful as biomarkers for early diagnosis and predictors of prognosis, and for monitoring response to therapies. Additionally, due to their role in the formation of CSCs and their maintenance, they could serve as novel targets for treating cancers by novel approaches focused on overcoming therapeutic resistance as discussed earlier.

\section{Different types of miRNAs involved in cancer}

Over 1100 miRNA sequences have been recognized in humans [60]. Some of these are deregulated in cancers. miRNAs are either oncogenic miRNAs or tumor suppressor miRNAs depending upon their effect on the tumors. Cellular signaling can be regulated by major miRNAs that have tumor suppressor or oncogenic activity. Oncogenic miRNAs are up-regulated in cancer, which down-regulates suppressor genes. The typical example of oncogenic miRNAs among others is miR21 which is over-expressed in cancers $\{$ Ali $7 / i d ;$ [61] Slaby, $20079 / \mathrm{id}\}$. Other oncogenic miRNAs include miR-17-92, miR-155, miR-221 and miR-222. Tumor suppressor miRNAs are down-regulated in cancer, resulting in the activation of oncogenes causing tumor aggressiveness. For example, let-7 family of miRNA is a tumor suppressor oncomiR which is under-expressed in malignancies [62-64]. Other tumor suppressor miRNAs include miR-15, miR-16, miR-17-5p, miR-29, miR-34, miR-124a, miR-127, miR-143, miR-145 and miR-181 among 
many others [65]. The list of specific miRNA that either functions as oncogenic or suppressor miRNAs is growing rapidly and it is becoming hard to keep-up with the rapid pace of our research advances.

\section{Alterations in the expression of miRNAs in cancer and therapeutic resistance}

It has been documented earlier in this article that altered expression of specific miRNAs is associated with therapeutic resistance. In docetaxel-resistant NSCLC cells, miR-192, miR-424 and miR-98 are over-expressed and miR-194, miR-200b and miR-212 are underexpressed [66]. Over-expression of miR-140 makes tumor cells more resistant to 5-FU [1]. Because deregulated miRNAs contribute to therapeutic resistance, miRNAs are attractive therapeutic targets for overcoming resistance. Thus, miRNAs are being used to restore the sensitivity of drug-resistant cells to chemotherapy and preventing tumor recurrence. For example, blocking endogenous miR-140 has been shown to sensitize resistant cancer cells to 5-FU treatment. Moreover re-expression of miR-200 increases the sensitivity to microtubuletargeting chemotherapeutic agents [67] whereas knock-down of miR221 and miR-222 has been shown to sensitize breast cancer cells to tamoxifen-induced apoptosis [68]. Another great example is that the suppression of miR-21 led to increased cytotoxicity of a semisynthetic podophyllotoxin derivative (VM-26) against glioblastoma cells [63]. These results are exciting and provide preliminary evidence that miRNAs could function as novel targets for overcoming therapeutic resistance (summarized in Figure 1).

\section{Clinical Relevance of miRNAs}

In normal development, miRNAs have an important physiologic role. The accumulation of let-7 miRNA is prevented by Lin28, a promoter of pluripotency [69] Pluripotency refers to the ability of stem cells to differentiate into any of the three germ layers: endoderm (interior stomach lining, gastrointestinal tract, the lungs), mesoderm (muscle, bone, blood, urogenital), or ectoderm (epidermal tissues and nervous system).In the similar context the cancer stem cells have the potential to undergo differentiation into the tumor cell type e.g. squamous or adenocarcinoma. The miRNA let-7 and Lin28 which maintain the delicate balance between pluripotency vs. differentiation during development may have potential use in tumor therapeutics as discussed further in this section.

Tumor specific miRNAs have potential clinical use as markers for accurate and early diagnosis, predicting prognosis and can serve as targets for future novel targeted therapies for malignancies as indicated above. Over-expression of oncogenic miRNAs and low expression of tumor suppressor miRNAs can serve as biomarkers for the identification of tumors either at the earliest stages of tumor development or in cases where there is a diagnostic dilemma. For example, miR-21, miR-1792, miR-155, miR-221 and miR-222 are over-expressed in cancers [61,62,70]whereas Let-7 [62-64], miR-15, miR-16, miR-17-5p, miR29, miR-34, miR-124a, miR-127, miR-143, miR-145 and miR-181 are under-expressed in malignancies [65].

Likewise, over-expression of oncogenic miRNAs and low expression of tumor suppressor miRNAs has been demonstrated to predict the clinical course of the disease in different cancers. It is well documented that cases in the same clinical stage do not always behave in the similar manner despite similar treatment protocols. Hence prognostic biomarkers are important to determine the survival of patients in the clinical context. Moreover, miR-21 over-expression predicts poor prognosis in breast cancers [71] and tumor aggressiveness in pancreatic cancers [62] whereas reduced expression of tumor suppressor oncomiR let-7 is associated with increased tumorigenicity and poor patient prognosis [69]. In breast cancer cells, reduced let-7 expression correlates with greater tumor forming capacity [72], and in NSCLC, it is associated with poor post-operative survival [64]. In ovarian cancer patients, reduced let-7 expression indicates shorter progression free survival [73]. The accumulation of let-7 miRNA is prevented by Lin28, a promoter of pluripotency [69]. Lin28B is a homolog of Lin28 that is over-expressed in many human cancers including prostate cancer (PCa), and is associated with advanced tumor stage [74]. Overexpression of Lin28B leads to the acquisition of invasive and metastatic capabilities in PCa cells. This happens via down-regulation of miR$200 \mathrm{~b}$ and miR-200c [74] or through repression of let-7. The repression of mature let-7 family leads to increased expression of EZH2 thereby maintaining the stem cell characteristics in PCa [74].

Cancers are a common cause of patient mortality and morbidity. This is mainly due to the lack of curative therapies and inherent complex heterogeneity of the tumors, making it difficult to predict which patient would respond to anticancer therapies. Therefore, exploiting the expression of miRNAs to identify which patients would benefit from targeted anticancer therapies provide a novel approach in the treatment of cancers. To that end, recent studies have proposed that the identification of plasma miRNA expression levels can be potentially used as molecular indicators of aggressive pancreatic cancer [75].

Reducing the expression of oncogenic miRNAs and enhancing the expression of tumor suppressor miRNA appears to be a novel approach in developing targeted pharmaco-therapies for cancer. Expression levels of oncogenic and tumor suppressor miRNAs can predict drug resistance in different tumors as well. For example, miR-21 overexpression predicts chemoresistance in pancreatic cancers [76,77] and drug resistance in glioblastomas [63] whereas in ovarian cancer reduced let-7 expression indicates resistance to chemotherapy [73]. In the next paragraphs we will discuss novel avenues by which one could deregulate the expression of miRNAs which could become a novel therapeutic strategy.

\section{Using miRNAs to overcome drug resistance}

Specific miRNAs can be targeted to overcome drug resistance and prevent tumor recurrence after chemotherapy. Re-expressing miR-200 increases the sensitivity to microtubule-targeting chemotherapeutic agents [67] whereas knock-down of miR-221 and miR-222 sensitizes breast cancer cells to tamoxifen-induced apoptosis [68]. Suppression of miR-21 also increases the cytotoxicity of a semisynthetic podophyllotoxin derivative, VM-26, against glioblastoma cells [63].

\section{CSC-targeted therapies and implication of developmental signaling}

Since targeting CSCs appears a promising therapeutic strategy for malignancies, several mechanisms have been postulated to destroy these self-renewing cells. For example, salinomycin reduces the proportion of CSCs by more than 100-fold when compared to paclitaxel, and inhibits mammary tumor growth in mice [78]. Moreover, Nigericin and abamectin can also inhibit the growth of CSCs [79]. The standard anti- diabetic drug, Metformin, selectively kills CSCs in breast cancer and when combined with doxorubicin killed both CSCs as well as nonCSCs [80]. In HER2-positive carcinomas, it acts synergistically with the anti-HER2 monoclonal antibody, trastuzumab, to suppress selfrenewal and proliferation of CSCs [81]. 
Isolating a pure CSC population for molecular analysis remains a challenge [6], and thus recent clinical trials have utilized developmental signaling pathways like Hedgehog [82], Notch and Wnt pathways and tested novel agents for the inhibition of these pathways to target CSCs $[83,84]$ without isolating CSCs. Recent studies are also targeting telomerase which is an important cell process for the maintenance of CSCs $[85,86]$. Another strategy is to induce terminal differentiation of the CSCs to modulate their function using interferon-alpha [87]. Interferon can eradicate CML CSCs in vitro unlike the drug imatinib which acts only against differentiated tumor cells and their progenitors [88]. Clinically, this is important as patients who receive imatinib alone relapse upon discontinuation of the drug [89]. Hence it has been recommended that to maintain molecular remission of the disease, CML patients should receive induction with both imatinib and interferon [90]. Since HER2/neu plays a role in breast CSCs selfrenewal [91], its inhibitor, lapatinib has been used in combination with cytotoxic agent capecitabine to increase the overall survival of breast cancer patients [92] However, targeted killing of CSCs by targeted activation or inactivation of miRNAs has been challenging.

P53, a tumor suppressor gene, transcriptionally activates microRNA miR-200c to regulate the EMT related CSC properties [93]. It directly binds to the miR-200c promoter. When p53 is lost, the expression of miR-200c is decreased resulting in activation of the EMT program accompanied by an increased cancer stem cell population. By re-expressing miR-200c we can suppress genes that mediate EMT and stemness properties and thereby revert the mesenchymal and stemcell-like phenotype caused by loss of p53 to a differentiated epithelial cell. This concept has relevance as a novel therapeutic approach for treating tumor aggressiveness.

\section{Role of Natural Agents in the Killing of CSCs by Targeting Mirnas}

Recently, natural agents have been shown to target CSCs in gemcitabine-resistant pancreatic cancer by up-regulation of miR-200 and let-7 [53]. Natural agents have the capacity to alter the expression of miRNAs (Figure 1) that are known to regulate cellular signaling and biological behavior in a safe manner with almost no toxicities (94). Curcumin, isoflavone, 3,3-diinodolylmethane (DIM), indole-3carbinol (I3C), epigallocatechin-3-gallate (EGCG) are typical examples of natural agents which have been demonstrated to regulate miRNAs [94-98]. DIM and isoflavone treatments has been shown to increase the level of miR-200 family in MiaPaCa-2 cells [53]. Curcumin upregulates the miR-22 expression [97]. Moreover, curcumin and piperine separately and in combination inhibited breast CSC self-renewal without causing toxicity to differentiated cells [99]. In pancreatic cancer, curcumin down-regulated the miR-21 and up-regulated the expression of miR-200 [75]. Indole-3-carbinol has been shown to down-regulate the expression of miR-21 and thereby up-regulates the miR-21 target gene PTEN in mouse lung tumors [96] whereas EGCG, another natural agent, up-regulates miR-16 expression in human tumor cells [98]. Sulforaphane derived from broccoli eradicates pancreatic CSCs-like cells in a synergistic manner when combined with the kinase inhibitor sorafenib [100]. Curcumin or its analogue $\mathrm{CDF}$ has been shown to induce gemcitabine sensitivity in pancreatic cancer cells by modulating miR-200 and miR-21 expression in our recent study [75]; summarized in Figure 1. In prostate cancer cells, miR-200 has been shown to regulate PDGF-D-mediated adhesion, invasion and epithelial-mesenchymal transition (EMT) [101], which was mechanistically linked with stem cell signatures [101,102] in prostate cancer cell model. Moreover, up-regulation of miR-146a has been shown to inhibit invasion of pancreatic cancer cells in our recent study [95]. Although natural agents could serve as an important agent for the deregulation of miRNAs, further in-depth research is certainly warranted.

\section{Perspectives and Concluding Remarks}

In conclusion, as a paradigm shift occurs in our understanding of CSCs, research continues in the field and the clinical relevance of the miRNAs continues to evolve in cancer diagnosis, prognosis as well as treatment. The role and therapeutic implications of miRNAs and a targeted approach in the context of CSCs and therapeutic resistance is certainly a promising and an active area of research (Figure 1), and their success is already on the horizon as we evaluate their use from the bench to bedside. Innovatively identifying CSC-specific hot spot oncogenic and tumor suppressor miRNAs have the potential to improve diagnostic accuracy, refine prognostic and predictive capabilities and serve as novel therapeutic targets. Elucidating the mechanisms by which miRNAs affect CSCs would fulfill an unmet need for understanding the pathobiology of CSCs in cancer recurrence, drug resistance and metastasis. Identifying clinically relevant CSC specific miRNA signatures would have potential translational application. It will help to develop a clinical algorithm, which will ultimately benefit the patients by improving diagnosis, predicting prognosis, and identifying patients who would benefit from anticancer therapies, those who would develop chemoresistance or those who already have de novo resistant cells to conventional therapeutics. Overall, it is our belief that in-depth mechanistic understanding of the role of miRNAs in CSCs would allow us to improve clinical decision making, which will further facilitate the discovery of novel therapies and therapeutic targets for personalized medicine.

\section{References}

1. Sarkar FH, Li Y, Wang Z, Kong D, Ali S (2010) Implication of microRNAs in drug resistance for designing novel cancer therapy. Drug Resist Updat 13: 57-66.

2. Gupta PB, Chaffer CL, Weinberg RA (2009) Cancer stem cells: mirage or reality? Nat Med 15: 1010-1012.

3. Clarke MF, Dick JE, Dirks PB, Eaves CJ, Jamieson CH, et al. (2006) Cancer stem cells--perspectives on current status and future directions: AACR Workshop on cancer stem cells. Cancer Res 66: 9339-9344.

4. Hamburger AW, Salmon SE (1977) Primary bioassay of human tumor stem cells. Science 197: 461-463.

5. Park CH, Bergsagel DE, McCulloch EA (1971) Mouse myeloma tumor stem cells: a primary cell culture assay. J Natl Cancer Inst 46: 411-422.

6. Rasheed ZA, Kowalski J, Smith BD, Matsui W (2011) Concise review: Emerging concepts in clinical targeting of cancer stem cells. Stem Cells 29: 883-887.

7. Lapidot T, Sirard C, Vormoor J, Murdoch B, Hoang T, et al. (1994) A cell initiating human acute myeloid leukaemia after transplantation into SCID mice. Nature 367: 645-648

8. Wang Z, Li Y, Banerjee S, Sarkar FH (2009) Emerging role of Notch in stem cells and cancer. Cancer Lett 279: 8-12.

9. Al-Hajj M, Wicha MS, Benito-Hernandez A, Morrison SJ, Clarke MF (2003) Prospective identification of tumorigenic breast cancer cells. Proc Natl Acad Sci U S A 100: 3983-3988.

10. Huff CA, Matsui W, Smith BD, Jones RJ (2006) The paradox of response and survival in cancer therapeutics. Blood 107: 431-434.

11. Ishikawa F, Yoshida S, Saito Y, Hijikata A, Kitamura H, et al. (2007) Chemotherapy-resistant human AML stem cells home to and engraft within the bone-marrow endosteal region. Nat Biotechnol 25: 1315-1321. 
Citation: Sethi S, Sarkar FH (2011) Evolving Concept of Cancer Stem Cells: Role of Micro-RNAs and their Implications in Tumor Aggressiveness. J Carcinogene Mutagene S1:005. doi:10.4172/2157-2518.S1-005

Page 6 of 8

12. Saito Y, Uchida N, Tanaka S, Suzuki N, Tomizawa-Murasawa M, et al. (2010) Induction of cell cycle entry eliminates human leukemia stem cells in a mouse model of AML. Nat Biotechnol 28: 275-80.

13. Lemoli RM, Salvestrini V, Bianchi E, Bertolini F, Fogli M, et al. (2009) Molecular and functional analysis of the stem cell compartment of chronic myelogenous leukemia reveals the presence of a CD34- cell population with intrinsic resistance to imatinib. Blood 114: 5191-5200.

14. Cammareri P, Scopelliti A, Todaro M, Eterno V, Francescangeli F, et al. (2010) Aurora-a is essential for the tumorigenic capacity and chemoresistance of colorectal cancer stem cells. Cancer Res 70: 4655-4665.

15. Fang DD, Kim YJ, Lee CN, Aggarwal S, McKinnon K, et al. (2010) Expansion of CD133(+) colon cancer cultures retaining stem cell properties to enable cancer stem cell target discovery. Br J Cancer 102: 1265-1275.

16. Ong CW, Kim LG, Kong HH, Low LY, lacopetta B, et al. (2010) CD133 expression predicts for non-response to chemotherapy in colorectal cancer. Mod Pathol 23: 450-457.

17. Liu G, Yuan X, Zeng Z, Tunici P, Ng H, et al. (2006) Analysis of gene expression and chemoresistance of CD133+ cancer stem cells in glioblastoma. Mol Cancer $5: 67$.

18. Zeppernick F, Ahmadi R, Campos B, Dictus C, Helmke BM, et al. (2008) Stem cell marker CD133 affects clinical outcome in glioma patients. Clin Cancer Res 14: $123-129$

19. Shi MF, Jiao J, Lu WG, Ye F, Ma D, et al. (2010) Identification of cancer stem cell-like cells from human epithelial ovarian carcinoma cell line. Cell Mol Life Sci 67: 3915-3925

20. Hu L, McArthur C, Jaffe RB (2010) Ovarian cancer stem-like side-population cells are tumourigenic and chemoresistant. Br J Cancer 102:1276-1283.

21. Wang B, Yang H, Huang YZ, Yan RH, Liu FJ, et al. (2010) Biologic characteristics of the side population of human small cell lung cancer cell line H446. Chin J Cancer 29: 254-260.

22. Gutova M, Najbauer J, Gevorgyan A, Metz MZ, Weng Y, et al. (2007) Identification of uPAR-positive chemoresistant cells in small cell lung cancer. PLoS One 2: e243.

23. Adhikari AS, Agarwal N, Wood BM, Porretta C, Ruiz B, et al. (2010) CD117 and Stro-1 identify osteosarcoma tumor-initiating cells associated with metastasis and drug resistance. Cancer Res 70: 4602-4012.

24. Hermann PC, Huber SL, Herrler T, Aicher A, Ellwart JW, et al. (2007) Distinct populations of cancer stem cells determine tumor growth and metastatic activity in human pancreatic cancer. Cell Stem Cell 1: 313-323.

25. Hong SP, Wen J, Bang S, Park S, Song SY (2009) CD44-positive cells are responsible for gemcitabine resistance in pancreatic cancer cells. Int J Cancer 125: 2323-2331.

26. Shafee N, Smith CR, Wei S, Kim Y, Mills GB, et al. (2008) Cancer stem cells contribute to cisplatin resistance in Brca1/p53-mediated mouse mammary tumors. Cancer Res 68: 3243-3250.

27. To K, Fotovati A, Reipas KM, Law JH, Hu K, et al. (2010) Y-box binding protein-1 induces the expression of CD44 and CD49f leading to enhanced selfrenewal, mammosphere growth, and drug resistance. Cancer Res 70: 28402851.

28. Zhang N, Li R, Tao KS, Cao DY, Ti ZY, et al. ( 2010) Characterization of a stem-like population in hepatocellular carcinoma MHCC97 cells. Oncol Rep 23: 827-831

29. Tokar EJ, Qu W, Liu J, Liu W, Webber MM, et al. (2010) Arsenic-specific stem cell selection during malignant transformation. J Natl Cancer Inst 102: 638-649.

30. Liu T, Xu F, Du X, Lai D, Liu T, et al. (2010) Establishment and characterization of multi-drug resistant, prostate carcinoma-initiating stem-like cells from human prostate cancer cell lines 22RV1. Mol Cell Biochem 340: 265-273.

31. Ginestier C, Hur MH, Charafe-Jauffret E, Monville F, Dutcher J, et al. (2007) ALDH1 is a marker of normal and malignant human mammary stem cells and a predictor of poor clinical outcome. Cell Stem Cell 1: 555-567.

32. Matsui W, Huff CA, Wang Q, Malehorn MT, Barber J, et al. (2004)
Characterization of clonogenic multiple myeloma cells. Blood 103: 2332-2336.

33. Singh SK, Hawkins C, Clarke ID, Squire JA, Bayani J, et al. (2004) Identification of human brain tumour initiating cells. Nature 432: 396-401.

34. Tang C, Ang BT, Pervaiz S (2007) Cancer stem cell: target for anti-cancer therapy. FASEB J 21: 3777-3785

35. Toole BP, Slomiany MG (2008) Hyaluronan, CD44 and Emmprin: partners in cancer cell chemoresistance. Drug Resist Updat 11: 110-121.

36. Liu T, Cheng W, Lai D, Huang Y, Guo L (2010) Characterization of primary ovarian cancer cells in different culture systems. Oncol Rep 23: 1277-1284.

37. Rasheed ZA, Yang J, Wang Q, Kowalski J, Freed I, et al. (2010) Prognostic significance of tumorigenic cells with mesenchymal features in pancreatic adenocarcinoma. J Natl Cancer Inst 102: 340-351.

38. Abraham BK, Fritz P, McClellan M, Hauptvogel P, Athelogou M, et al. (2005) Prevalence of CD44+/CD24-/low cells in breast cancer may not be associated with clinical outcome but may favor distant metastasis. Clin Cancer Res 11 : 1154-1159.

39. Liu R, Wang X, Chen GY, Dalerba P, Gurney A, et al. (2007) The prognostic role of a gene signature from tumorigenic breast-cancer cells. N Engl J Med 356: 217-226.

40. Shipitsin M, Campbell LL, Argani P, Weremowicz S, Bloushtain-Qimron N, et al. (2007) Molecular definition of breast tumor heterogeneity. Cancer Cell 11 259-273.

41. Diehn M, Cho RW, Lobo NA, Kalisky T, Dorie MJ, et al. (2009) Association of reactive oxygen species levels and radioresistance in cancer stem cells. Nature 458: 780-783.

42. Bao S, Wu Q, McLendon RE, Hao Y, Shi Q, et al. (2006) Glioma stem cells promote radioresistance by preferential activation of the DNA damage response. Nature 444: 756-760.

43. Li X, Lewis MT, Huang J, Gutierrez C, Osborne CK, et al. (2008) Intrinsic resistance of tumorigenic breast cancer cells to chemotherapy. J Natl Cancer Inst 100: 672-679.

44. Dylla SJ, Beviglia L, Park IK, Chartier C, Raval J, et al. (2008) Colorectal cance stem cells are enriched in xenogeneic tumors following chemotherapy. PLoS One 3: e2428.

45. Matsui W, Wang Q, Barber JP, Brennan S, Smith BD, et al. (2008) Clonogenic multiple myeloma progenitors, stem cell properties, and drug resistance. Cancer Res 68: 190-197.

46. Brennan SK, Meade B, Wang Q, Merchant AA, Kowalski J, et al. (2010) Mantle cell lymphoma activation enhances bortezomib sensitivity. Blood 116: 41854191.

47. Graham SM, Jørgensen HG, Allan E, Pearson C, Alcorn MJ, et al. (2002) Primitive, quiescent, Philadelphia-positive stem cells from patients with chronic myeloid leukemia are insensitive to STI571 in vitro. Blood 99: 319-325.

48. Dean M, Fojo T, Bates S (2005) Tumour stem cells and drug resistance. Nat Rev Cancer 5:275-284

49. Styczynski J, Drewa T (2007) Leukemic stem cells: from metabolic pathways and signaling to a new concept of drug resistance targeting. Acta Biochim Pol 54: 717-1726.

50. Zhou S, Schuetz JD, Bunting KD, Colapietro AM, Sampath J, et al. (2001) The $A B C$ transporter Bcrp1/ABCG2 is expressed in a wide variety of stem cells and is a molecular determinant of the side-population phenotype. Nat Med 7: 10281034.

51. Ventura A, Jacks T (2009) MicroRNAs and cancer: short RNAs go a long way. Cell 136: 586-91.

52. Vandenboom li TG, Li Y, Philip PA, Sarkar FH (2008) MicroRNA and Cancer: Tiny Molecules with Major Implications. Curr Genomics 9: 97-109.

53. Li Y, VandenBoom TG, Kong D, Wang Z, Ali S, et al. (2009) Up-regulation of miR-200 and let-7 by natural agents leads to the reversal of epithelial-tomesenchymal transition in gemcitabine-resistant pancreatic cancer cells. Cancer Res 69: 6704-6712. 
Citation: Sethi S, Sarkar FH (2011) Evolving Concept of Cancer Stem Cells: Role of Micro-RNAs and their Implications in Tumor Aggressiveness. J Carcinogene Mutagene S1:005. doi:10.4172/2157-2518.S1-005

Page 7 of 8

54. Shimono Y, Zabala M, Cho RW, Lobo N, Dalerba P, et al. (2009) Downregulation of miRNA-200c links breast cancer stem cells with normal stem cells. Cell 138 : $592-603$

55. Shi L, Zhang J, Pan T, Zhou J, Gong W, et al. (2010) MiR-125b is critical for the suppression of human U251 glioma stem cell proliferation. Brain Res 1312: $120-126$

56. Wellner U, Schubert J, Burk UC, Schmalhofer O, Zhu F, et al. (2009) The EMTactivator ZEB1 promotes tumorigenicity by repressing stemness-inhibiting microRNAs. Nat Cell Biol 11: 1487-1495.

57. Ji Q, Hao X, Zhang M, Tang W, Yang M, et al. (2009) MicroRNA miR-34 inhibits human pancreatic cancer tumor-initiating cells. PLoS One 4: e6816.

58. Yu F, Deng H, Yao H, Liu Q, Su F, Song E (2010) Mir-30 reduction maintains self-renewal and inhibits apoptosis in breast tumor-initiating cells. Oncogene 29: 4194-4204

59. Nicoloso MS, Spizzo R, Shimizu M, Rossi S, Calin GA (2009) MicroRNAs--the micro steering wheel of tumour metastases. Nat Rev Cancer 9: 293-302.

60. Griffiths-Jones S, Saini HK, van Dongen S, Enright AJ (2008) miRBase: tools for microRNA genomics. Nucleic Acids Res 36: D154-D158.

61. Resnick KE, Alder H, Hagan JP, Richardson DL, Croce CM, et al. (2009) The detection of differentially expressed microRNAs from the serum of ovarian cancer patients using a novel real-time PCR platform. Gynecol Oncol 112: 5559.

62. Ali S, Ahmad A, Banerjee S, Padhye S, Dominiak K, et al. (2010) Gemcitabine sensitivity can be induced in pancreatic cancer cells through modulation of $\mathrm{miR}-200$ and miR-21 expression by curcumin or its analogue CDF. Cancer Res 70: $3606-3617$

63. Li Y, Li W, Yang Y, Lu Y, He C , et al. (2009) MicroRNA-21 targets LRRFIP1 and contributes to VM-26 resistance in glioblastoma multiforme. Brain Res 1286: $13-18$

64. Takamizawa J, Konishi H, Yanagisawa K, Tomida S, Osada H, et al. (2004) Reduced expression of the let-7 microRNAs in human lung cancers in association with shortened postoperative survival. Cancer Res 64: 3753-3756.

65. Croce CM. (2009) Causes and consequences of microRNA dysregulation in cancer. Nat Rev Genet 10: 704-714.

66. Rui W, Bing F, Hai-Zhu S, Wei D, Long-Bang C (2010) Identification of microRNA profiles in docetaxel-resistant human non-small cell lung carcinoma cells (SPC-A1). J Cell Mol Med 14: 206-214.

67. Cochrane DR, Spoelstra NS, Howe EN, Nordeen SK, Richer JK (2009) MicroRNA-200c mitigates invasiveness and restores sensitivity to microtubuletargeting chemotherapeutic agents. Mol Cancer Ther 8: 1055-66.

68. Zhao JJ, Lin J, Yang H, Kong W, He L, et al. (2008) MicroRNA-221/222 negatively regulates estrogen receptor alpha and is associated with tamoxifen resistance in breast cancer. J Biol Chem 283: 31079-31086.

69. Bussing I, Slack FJ, Grosshans H (2008) let-7 microRNAs in development, stem cells and cancer. Trends Mol Med 14: 400-409.

70. Slaby O, Svoboda M, Fabian P, Smerdova T, Knoflickova D, et al. (2007) Altered expression of miR-21, miR-31, miR-143 and miR-145 is related to clinicopathologic features of colorectal cancer. Oncology 72: 397-402.

71. Shi M, Guo N (2009) MicroRNA expression and its implications for the diagnosis and therapeutic strategies of breast cancer. Cancer Treat Rev 35: 328-334.

72. Yu F, Yao H, Zhu P, Zhang X, Pan Q, et al. (2007) let-7 regulates self renewal and tumorigenicity of breast cancer cells. Cell 131: 1109-1123.

73. Yang N, Kaur S, Volinia S, Greshock J, Lassus H, et al. (2008) MicroRNA microarray identifies Let-7i as a novel biomarker and therapeutic target in human epithelial ovarian cancer. Cancer Res 68: 10307-10314.

74. Kong D, Banerjee S, Ahmad A, Li Y, Wang Z, et al. (2010) Epithelial to mesenchymal transition is mechanistically linked with stem cell signatures in prostate cancer cells. PLoS One 5: e12445.

75. Ali S, Almhanna K, Chen W, Philip PA, Sarkar FH (2010) Differentially expressed miRNAs in the plasma may provide a molecular signature for aggressive pancreatic cancer. Am J Transl Res 3: 28-47.

76. Moriyama T, Ohuchida K, Mizumoto K, Yu J, Sato N, et al. (2009) MicroRNA-21 modulates biological functions of pancreatic cancer cells including thei proliferation, invasion, and chemoresistance. Mol Cancer Ther 8: 1067-1074.

77. Park JK, Lee EJ, Esau C, Schmittgen TD (2009) Antisense inhibition of microRNA-21 or -221 arrests cell cycle, induces apoptosis, and sensitizes the effects of gemcitabine in pancreatic adenocarcinoma. Pancreas 38: e190-e199.

78. Gupta PB, Onder TT, Jiang G, Tao K, Kuperwasser C, et al. (2009) Identification of selective inhibitors of cancer stem cells by high-throughput screening. Cell 138: 645-659.

79. Riccioni R, Dupuis ML, Bernabei M, Petrucci E, Pasquini L, et al. (2010) The cancer stem cell selective inhibitor salinomycin is a p-glycoprotein inhibitor Blood Cells Mol Dis 45: 86-92.

80. Hirsch HA, Iliopoulos D, Tsichlis PN, Struhl K (2009) Metformin selectively targets cancer stem cells, and acts together with chemotherapy to block tumor growth and prolong remission. Cancer Res 69: 7507-7511.

81. Vazquez-Martin A, Oliveras-Ferraros C, Barco SD, Martin-Castillo B, Menendez JA, et al. (2011) The anti-diabetic drug metformin suppresses self-renewal and proliferation of trastuzumab-resistant tumor-initiating breast cancer stem cells. Breast Cancer Res Treat 126: 355-364.

82. Rudin CM, Hann CL, Laterra J, Robert L. Yauch, Christopher A. Callahan, et al. (2009) Treatment of medulloblastoma with hedgehog pathway inhibitor GDC 0449. N Engl J Med 361: 1173-1178.

83. Bar EE, Chaudhry A, Lin A, Fan X, Schreck K, et al. (2007) Cyclopaminemediated hedgehog pathway inhibition depletes stem-like cancer cells in glioblastoma. Stem Cells 25: 2524-2533.

84. Li BZ, Shi MX, Li J, Ma J, Chen L, et al. (2009) [The effect of TNF-alpha on TAZ expression and osteogenic potential of mesenchymal stem cells from myeloma patients]. Zhonghua Zhong Liu Za Zhi 31: 5-9.

85. Brennan SK, Wang Q, Tressler R, Harley C, Go N, et al. (2010) Telomerase inhibition targets clonogenic multiple myeloma cells through telomere lengthdependent and independent mechanisms. PLoS One 5: e12487.

86. Marian CO, Cho SK, McEllin BM, Maher EA, Hatanpaa KJ, et al. (2010) The telomerase antagonist, imetelstat, efficiently targets glioblastoma tumorinitiating cells leading to decreased proliferation and tumor growth. Clin Cancer Res 16: 154-163.

87. Matsui W, Huff CA, Vala M, Barber J, Smith BD, et al. (2003) Anti-tumour activity of interferon-alpha in multiple myeloma: role of interleukin 6 and tumor cell differentiation. $\mathrm{Br} \mathrm{J}$ Haematol 121: 251-258.

88. Angstreich GR, Matsui W, Huff CA, Vala MS, Barber J, et al. (2005) Effects of imatinib and interferon on primitive chronic myeloid leukaemia progenitors. $\mathrm{Br}$ Haematol 130: 373-381.

89. Rousselot P, Huguet F, Rea D, Legros L, Cayuela JM, et al. (2007) Imatinib mesylate discontinuation in patients with chronic myelogenous leukemia in complete molecular remission for more than 2 years. Blood 109: 58-60.

90. Burchert A, Muller MC, Kostrewa P, Erben P, Bostel T, et al. (2010) Sustained molecular response with interferon alfa maintenance after induction therapy with imatinib plus interferon alfa in patients with chronic myeloid leukemia. $J$ Clin Oncol 28: 1429-1435.

91. Korkaya H, Paulson A, lovino F, Wicha MS (2008) HER2 regulates the mammary stem/progenitor cell population driving tumorigenesis and invasion. Oncogene 27: 6120-6130.

92. Geyer CE, Forster J, Lindquist D, Stephen Chan, Gilles Romieu C, et al. (2006) Lapatinib plus capecitabine for HER2-positive advanced breast cancer. N Eng J Med 355: 2733-2743.

93. Chang CJ, Chao CH, Xia W, Yang JY, Xiong Y, et al. (2011) p53 regulates epithelial-mesenchymal transition and stem cell properties through modulating miRNAs. Nat Cell Biol 13: 317-323.

94. Li Y, Kong D, Wang Z, Sarkar FH (2010) Regulation of microRNAs by natura agents: an emerging field in chemoprevention and chemotherapy research Pharm Res 27: 1027-1041. 
Citation: Sethi S, Sarkar FH (2011) Evolving Concept of Cancer Stem Cells: Role of Micro-RNAs and their Implications in Tumor Aggressiveness. J Carcinogene Mutagene S1:005. doi:10.4172/2157-2518.S1-005

Page 8 of 8

95. Li Y, VandenBoom TG, Wang Z, Kong D, Ali S, et al. ( 2010) miR-146a suppresses invasion of pancreatic cancer cells. Cancer Res 70: 1486-1495.

96. Melkamu T, Zhang X, Tan J, Zeng Y, Kassie F ( 2010) Alteration of microRNA expression in vinyl carbamate-induced mouse lung tumors and modulation by the chemopreventive agent indole-3-carbinol. Carcinogenesis 31: 252-258

97. Sun M, Estrov Z, Ji Y, Coombes KR, Harris DH, et al. (2008) Curcumin (diferuloylmethane) alters the expression profiles of microRNAs in human pancreatic cancer cells. Mol Cancer Ther 7: 464-473.

98. Tsang WP, Kwok TT (2010) Epigallocatechin gallate up-regulation of miR-16 and induction of apoptosis in human cancer cells. J Nutr Biochem 21: 140-146.

99. Kakarala M, Brenner DE, Korkaya H, Connie Cheng, Karim Tazi, et al. ( 2010)
Targeting breast stem cells with the cancer preventive compounds curcumin and piperine. Breast Cancer Res Treat 122: 777-785.

100. Rausch V, Liu L, Kallifatidis G, Baumann B, Mattern J, et al. (2010) Synergistic activity of sorafenib and sulforaphane abolishes pancreatic cancer stem cel characteristics. Cancer Res 70: 5004-5013.

101. Kong D, Li Y, Wang Z, Banerjee S, Ahmad A, et al. (2009) miR-200 regulates PDGF-D-mediated epithelial-mesenchymal transition, adhesion, and invasion of prostate cancer cells. Stem Cells 27: 1712-1721.

102. Sethi S, Macoska J, Chen W, Sarkar FH (2010) Molecular signature of epithelial-mesenchymal transition (EMT) in human prostate cancer bone metastasis. Am J Transl Res 3: 90-99.
This article was originally published in a special issue, Stem Cells-Cancer Research handled by Editor(s). Dr. Claudio Luparello, University of Palermo, Italy 